\title{
Fe y razón en la filosofía católica: la propuesta de Alasdair MacIntyre
}

\author{
CRISTÓBAL ORREGO SÁNCHEZ \\ Pontificia Universidad Católica de Chile (Chile) \\ corregos@uc.cl
}

\begin{abstract}
Resumen
Tras un breve recuerdo del debate centenario sobre la filosofía cristiana, el autor discute la nueva propuesta de Alasdair MacIntyre de mejorar una «filosofía católica» en el contexto de nuestra moderna crisis en la relación entre fe y razón. El autor comparte el diagnóstico sobre nuestra situación presente en las universidades de investigación y sobre su exclusión pragmática de la filosofía y la teología, las cuales, en el mejor caso, son reducidas a disciplinas especializadas sin relevancia para otras disciplinas, y, en la mayoría de los casos, son simplemente expulsadas del ámbito de la investigación científica. La propuesta dialéctica de MacIntyre en Tres versiones rivales de la ética y en Dios, Filosofía, Universidades, aparte de otras obras menores, se examina críticamente y se presenta como una forma nueva de comprometer a los pensadores católicos y no católicos en una confrontación abierta y fructífera, en lugar de nuestro confinamiento actual en mundos cerrados de investigación falsamente científica.
\end{abstract}

Palabras clave: Fe y razón, filosofía católica (cristiana), Universidad, MacIntyre.

\section{Faith and reason in Catholic philosophy: Alasdair MacIntyre's proposal}

\begin{abstract}
After a brief recollection of the century long debate about Christian philosophy, the author discusses Alasdair MacIntyre's new proposal to enhance a "Catholic philosophy» in the context of our modern crisis of the relationship between faith and reason. The author shares the diagnosis about our present day situation of research universities and their pragmatic exclusion of philosophy and theology, which, in the best case, are reduced to specialized disciplines with no import for other disciplines, and, in most cases, are just expelled from the realm of scientific research. MacIntyre's dialectical proposal in "Three Rival Versions of Moral Enquiry» and in "God, Philosophy, Universities», and in some other minor works, is critically examined and presented as a new way to engage Catholic and non Catholic thinkers in an open and fruitful confrontation, in stead of our present confinement in closed worlds of falsely scientific research.
\end{abstract}

Key words: Faith and Reason, Catholic (Christian) philosophy, University, MacIntyre.

Licenciado en Derecho por la Pontificia Universidad Católica de Chile, Abogado, Máster en Artes Liberales y Doctor en Derecho por la Universidad de Navarra. Profesor de Filosofía del Derecho en la Facultad de Derecho de la PUC. Este artículo procede de en una ponencia en las Reuniones Filosóficas de la Universidad de Navarra, octubre 9-12, 2013. Agradezco a los organizadores la oportunidad de discutirla y de mejorarla, que me han brindado. Doy las gracias, además, a la Pontificia Universidad Católica de Chile por la ayuda a la investigación en que se enmarca esta reflexión: Proyecto de Investigación "Las universidades católicas en el nuevo milenio: identidad y misión a medio siglo del Concilio Vaticano II” (DPCC 1623/2012). 


\section{Introducción}

Jean Guitton dedica su Testamento Filosófico (1998) a narrar unas conversaciones imaginarias, en su lecho de muerte, con personajes tan diversos como Lucifer, Pascal y el Papa Pablo VI. Todos los temas abordados son, muy apropiadamente, de vida o muerte: la verdad y la actitud crítica, la fe y la razón, el amor y la hipocresía... Sin duda que se trata de un testamento, es decir, de aquello que, a la luz de la muerte, se quiere dejar como últimas palabras, $y$, en consecuencia, como esas que uno estima fundamentales; pero, ¿por qué llamarlo «filosófico»? ¿Acaso no trata de cuestiones eminentemente religiosas y acaso no omite la profundidad, el rigor y el detalle de las argumentaciones racionales, bajo la protección de una narrativa ingeniosa y de la indulgencia con que juzgamos a los difuntos? ¿Qué tiene de filosófico el testamento filosófico de Jean Guitton?

John Finnis, en su reciente opúsculo Ruptura, transformación y continuidad en la tradición de la razón y la justicia (Finnis, 2013), dedica la mayor parte de su esfuerzo a defender el carácter racional de una tradición cuyas tesis fundamentales surgieron de la revelación divina, aunque sean comprensibles y aun demostrables racionalmente. De paso, Finnis sostiene que autores ateos como Herbert Hart y Ronald Dworkin nunca explicitaron los presupuestos profundos de sus escritos académicos, excepto Dworkin hacia el final de su vida, cuando defiende una religión sin Dios. ¿Cuáles eran esos presupuestos? En síntesis, ellos se reducen al rechazo de Dios y de las convicciones fundamentales sobre la creación, la providencia y, especialmente, la libertad humana (Finnis, 2013: 43). Dworkin, en Religion Without God, obra póstuma con las tres Einstein Lectures que impartió en la Universidad de Berna en el 2011, llega a afirmar un determinismo tan radical que declara como absolutamente necesario incluso el que él mismo llegara a existir (Dworkin, 2013: 82-103).

John Finnis hace lo contrario, más en la línea de Jean Guitton: explicita esas convicciones básicas. Tras mostrar su origen histórico en una revelación divina, sostiene que es razonable aceptarlas tras un examen racional riguroso. El examen racional de esa revelación y la intrínseca superioridad de sus contenidos fundamentales, en comparación con los presupuestos de otras tradiciones culturales y filosóficas ( $v . g r$., el ateísmo, el empirismo y el positivismo, que durante algún tiempo el mismo Finnis defendió), permiten abrazar la fe católica sin dar un salto en el vacío, sin entregarse a un mero impulso ciego del espíritu (Finnis, 2013).

Alasdair MacIntyre, quien, como Finnis, se convirtió del ateísmo al catolicismo tras un proceso de indagación racional, y que ahora se halla en el tramo último de su vida terrena, ha dedicado varias conferencias, seminarios y cursos, y su libro God, Philosophy, Universities, a establecer un 
contraste neto entre la filosofía secularizada y la filosofía católica (MacIntyre, 2009). Su tesis de las tres versiones rivales de investigación moral (i.e., la Ilustración, la genealogía de la moral de Nietzsche y el tomismo revivido gracias al impulso de la Encíclica Aeterni Patris) parece haberse reducido a una sola contraposición radical: la filosofía católica versus la filosofía secularizada. Es algo así como esos partidos de fútbol donde el equipo campeón se bate contra el resto del mundo. En este caso, la filosofía católica se confronta con un solo rival, representado por todas las filosofías que se han apartado del influjo benéfico de la fe. El contraste no se basa en cuestiones de detalle, ni se detiene en coincidencias menores entre autores católicos y no católicos, sino que, una vez más, se refiere a las preguntas fundamentales de la filosofía, que son las que inquietan a un ser humano en cuanto humano y solo por eso son asumidas como las cuestiones últimas por el filósofo que comprende adecuadamente su tarea como servicio al bien común (MacIntyre, 2009: 11): quién es el hombre, cómo se relaciona con la realidad, cómo procede de Dios y a Dios se dirige...

Podríamos buscar otros ejemplos de filósofos que, hacia el final de sus vidas, después de décadas de participar de un sistema académico que, como el nuestro occidental, cercena metódicamente los intentos de centrar la filosofía en aquello que es relevante para todos, retornan como voces aisladas a eso que verdaderamente importa. Nos contentaremos, no obstante, con proponer brevemente un diagnóstico de la situación y una propuesta, apoyándonos en una lectura crítica del pensamiento de Alasdair MacIntyre. Sin embargo, antes de centrar nuestro discurso en MacIntyre, nos parece conveniente situar su diagnóstico (sección 3) y su propuesta (sección 4) en el marco de una sucinta discusión de la controversia sobre la posibilidad de una filosofía cristiana (sección 2), que MacIntyre asume bajo la denominación de «filosofía católica».

\section{La controversia centenaria sobre la filosofía cristiana}

La expresión filosofía cristiana se hizo corriente en el debate de comienzos del siglo XX, especialmente en el ámbito cultural francés, a partir del momento en que Emile Bréhier, en 1927, afirmó, sin más, que no bay una filosofía cristiana. Le respondieron diversos autores, entre ellos Gilson, Chenu, Maritain y varios otros. En el marco de esa controversia, cabe distinguir al menos dos cuestiones diversas, aunque relacionadas. Por una parte, se discute una tesis histórica; por otra, una cuestión conceptual. El debate histórico comenzó con la tesis de Bréhier, según el cual no hay una filosofía cristiana en el sentido de que el pensamiento filosó- 
fico no ba sido fuertemente influenciado por el cristianismo (Derisi, 1979: 15). Bréhier concluye, de sus observaciones históricas, lo siguiente:

Se ve, pues, lo que absorbe el cristianismo de la cultura helénica: materiales para la gran obra religiosa de salvación del hombre; del espíritu que animaba a aquella no parece tener la más leve idea. No se trata de entenderla por dentro, sino sólo de inventariarla y utilizarla (Bréhier, 1988: 436).

En suma, para Bréhier la relación entre filosofía y cristianismo es meramente instrumental. Esta tesis meramente histórica nos parece haber sido refutada por Etienne Gilson en El Espiritu de la Filosofía Medieval, en un sentido trivial, pues constata el influjo histórico de ideas cristianas en la filosofía, incluyendo el claramente perceptible en aquellas filosofías incompatibles con el catolicismo (v.gr., las de Hegel y Marx) (Gilson, 2009: 15-16). Naturalmente, no se trataba de eso tan solo. La tesis histórica de un simple influjo del cristianismo en la especulación filosófica no era lo que realmente se quería decir cuando se destacaba una filosofía cristiana respecto de sus alternativas. La cuestión conceptual o doctrinal sobre en qué puede consistir una filosofía cristiana en cuanto tal es independiente de la cuestión histórica, y la condiciona, porque, en efecto, «si bajo la faz doctrinaria se resuelve el pleito en el sentido de la imposibilidad de una tal filosofía, es evidente que ella no ha podido existir» (Derisi, 1979: 18).

El mismo Gilson, que defiende una noción de filosofía cristiana y su existencia histórica, afirma que, si consideramos «la filosofía en sí misma, en su esencia formal y haciendo abstracción de las condiciones que presiden tanto su constitución como su inteligibilidad», en ese sentido «está claro que una filosofía no podría ser cristiana, ni tampoco judía o musulmana; y que la noción de filosofía cristiana no tiene mayor sentido que la de física o matemática cristiana» (Gilson, 2009: 41). La filosofía cristiana —según el mismo autor- es algo más que aquella compatible con la revelación y que la influida por ideas cristianas: es «toda filosofía que, aun cuando haga la distinción formal de los dos órdenes, considere la revelación cristiana como un auxiliar indispensable de la razón» (Gilson, 2009: 41).

Jacques Maritain propuso, por su parte, distinguir entre la naturaleza de la filosofía, que en cuanto tal no es cristiana, porque su objeto es accesible a la razón sin ayuda de la gracia, y su estado concreto o existencial, el de las condiciones reales de su ejercicio, donde sí que cabe hablar de una filosofía cristiana. En efecto, la revelación cristiana crea un nuevo régimen para la inteligencia bumana tanto porque le ofrece aportaciones objetivas o de contenido (v.gr., verdades naturales no descubiertas antes, co- 
mo la creación, el ser divino como acto mismo de ser subsistente, etc., y mayor certeza sobre verdades conocidas de manera probable o dudosa) (Maritain, 1933: 39-48) como porque influye en el aspecto subjetivo, en el hábito y en el ejercicio mismo del filosofar por quien filosofa (v.gr., influjo de la gracia, que alienta la actividad filosófica; cura de la presunción, al poner a la filosofía en su lugar bajo una sabiduría superior; seguridad o certeza respecto del origen y destino sobrenatural del hombre) (Maritain, 1933: 48-54).

En definitiva se comprende que no es solamente por el lado de los objetos propuestos, sino también por el lado de la vitalidad de la inteligencia y de sus más profundas inspiraciones como el estado de la filosofía ha sido cambiado y elevado por el cristianismo. Es según todos estos títulos que hay que decir que la fe guía y orienta a la filosofía, veluti stella rectrix, sin lesionar por ello su autonomía, pues es siempre según sus leyes propias y sus principios propios y en virtud de los meros criterios racionales como la filosofía juzga de las cosas; incluso de esas cosas que, aunque naturalmente accesibles a la mera razón, no serían de hecho reconocidas o mantenidas sin mezcla de error por la razón si, a su vez, esta no hubiese sido hecha atenta a su existencia y hecha más fuerte ella misma por una especie de continuidad vital con luces superiores (Maritain, 1933: 54; traducción, con ligera variante, de Palacios, 2010: 7).

A Maritain, Gilson y la mayoría de los autores, se opuso la Escuela de Lovaina, fundada por el Cardenal Mercier. Fernand van Steenberghen, poco antes de su muerte, sintetizó su posición, la posición de esta escuela, que rehúsa hablar de filosofía cristiana. Ciertamente se acepta que, si se entiende por filosofía en sentido amplio una cierta concepción del mundo (Weltanschaunng), existe una tal cosmovisión cristiana (van Steenberghen, 1993: 1088-1089). Tampoco se trata de negar un influjo del cristianismo en la filosofía en sentido estricto, como ciencia o saber metódico y reflexivo, pero ha sido un influjo tanto positivo como negativo (van Steenberghen, 1993: 1091). Sin embargo, en este sentido estricto o científico no debe hablarse de filosofía cristiana, «porque la influencia del cristianismo no puede intervenir jamás como tal en la elaboración de la filosofía, que debe construirse exclusivamente sobre la base de la experiencia con la ayuda de métodos racionales» (van Steenberghen, 1993: 1089). Además,

la influencia de las doctrinas cristianas actúa sobre la persona del filósofo cristiano: él es quien se beneficia de las luces de la revelación, ésta le ofrece conocimientos nuevos, más o menos misteriosos, que estimulan su reflexión, le sugieren problemas nuevos, le ponen en guardia contra graves errores; 
esta nueva visión le pone en las mejores condiciones psicológicas para hacer buena filosofía; pero su filosofía no será nunca "cristiana" ni respeta los métodos esenciales de la filosofía (van Steenberghen, 1993: 1089).

A mi modo de ver, no obstante, si la principal razón para rechazar que «una filosofía en sentido estricto sea calificada de cristiana» es que «la naturaleza de esta filosofía excluye esta calificación», aunque «es posible, legítimo y deseable que los pensadores cristianos se esfuercen en elaborar filosofías auténticas» (van Steenberghen, 1993: 1090), el desacuerdo no es tan radical, pues Gilson y Maritain también afirman que, desde la perspectiva abstracta — no existencial, ni histórica—, la filosofía no admite ese apellido.

Van Steenberghen tiene, sin embargo, una segunda razón para oponerse al uso de la expresión «filosofía cristiana».

A esta razón fundamental de evitar la fórmula filosófica cristiana [sic] se añade otra, a la que concedemos una importancia capital: la filosofía es un dominio del saber en el que es posible una colaboración intelectual entre creyentes y no creyentes. Esta colaboración es evidentemente un bien: preserva al creyente del asilamiento intelectual y abre a los no creyentes una vía que puede conducirles al conocimiento de Dios y al cristianismo (van Steenberghen, 1993: 1090).

Curiosamente, se trata de una motivación práctica religiosa: «facilitar el diálogo con los no creyentes» (van Steenberghen, 1993: 1092) y evitar así encerrar a los cristianos «en un ghetto intelectual sosteniendo que están condenados a pensar de manera distinta a los demás, incluso cuando hacen filosofía» (van Steenberghen, 1993: 1092). Esta motivación revela, a mi modo de ver, el verdadero problema de nuestro tiempo: mirar como una carga lo que, en realidad, es una fuente de superioridad; como una condena, precisamente, aquello que constituye la salvación de la razón por la ayuda de la fe, gracias a una razón humana que se abre más allá de sí misma. El Papa Benedicto XVI lo expresa así:

Sin duda, la naturaleza específica de la fe es la relación con el Dios vivo, un encuentro que nos abre nuevos horizontes mucho más allá del ámbito propio de la razón. Pero, al mismo tiempo, es una fuerza purificadora para la razón misma. Al partir de la perspectiva de Dios, la libera de su ceguera y la ayuda así a ser mejor ella misma. La fe permite a la razón desempeñar del mejor modo su cometido y ver más claramente lo que le es propio (Benedicto XVI, 2005: n. 28). 
Ya antes, Josef Ratzinger afirmaba la apertura de la razón a la fe, entre otras formas, así:

Es importante darles voz en el intento de una auténtica correlación polifónica en la que se abran a la esencial relación complementaria de razón y fe, de modo que pueda crecer un proceso universal de purificación en el que al final puedan resplandecer de nuevo los valores y las normas que en cierto modo todos los hombres conocen o intuyen, y así pueda adquirir nueva fuerza efectiva entre los hombres lo que cohesiona al mundo (Ratzinger, 2008: 54).

Juan Pablo II expuso su opinión, muy matizada, en su Encíclica Fides et ratio, especialmente en el n. 76 . Entiende la filosofía cristiana como «un modo de filosofar cristiano, una especulación filosófica concebida en unión vital con la fe» y no «como una filosofía oficial de la Iglesia, puesto que la fe como tal no es una filosofía» (Juan Pablo II, 1998: n. 76). Él no adopta - ni fuerza a adoptar- dicha expresión, con lo cual no interviene para zanjar el debate filosófico sobre la filosofía cristiana. El Papa constata el hecho de que muchos usan la denominación, y admite que es legítimo hacerlo siempre que se eviten malas interpretaciones. Vale la pena atender al pasaje completo (véase una explicación detallada de este pasaje en García López, 1999: 644-650):

Una segunda posición de la filosofía es la que muchos designan con la expresión filosofía cristiana. La denominación es en sí misma legítima, pero no debe ser mal interpretada: con ella no se pretende aludir a una filosofía oficial de la Iglesia, puesto que la fe como tal no es una filosofía. Con este apelativo se quiere indicar más bien un modo de filosofar cristiano, una especulación filosófica concebida en unión vital con la fe. No se hace referencia simplemente, pues, a una filosofía hecha por filósofos cristianos, que en su investigación no han querido contradecir su fe. Hablando de filosofía cristiana se pretende abarcar todos los progresos importantes del pensamiento filosófico que no se hubieran realizado sin la aportación, directa o indirecta, de la fe cristiana.

Dos son, por tanto, los aspectos de la filosofía cristiana: uno subjetivo, que consiste en la purificación de la razón por parte de la fe. Como virtud teologal, la fe libera la razón de la presunción, tentación típica a la que los filósofos están fácilmente sometidos. Ya san Pablo y los Padres de la Iglesia y, más cercanos a nuestros días, filósofos como Pascal y Kierkegaard la han estigmatizado. Con la humildad, el filósofo adquiere también el valor de afrontar algunas cuestiones que difícilmente podría resolver sin considerar los datos recibidos de la Revelación. Piénsese, por ejemplo, en los problemas del mal y del sufrimiento, en la identidad personal de Dios y en la pre- 
gunta sobre el sentido de la vida o, más directamente, en la pregunta metafísica radical: ¿Por qué existe algo?

Además está el aspecto objetivo, que afecta a los contenidos. La Revelación propone claramente algunas verdades que, aun no siendo por naturaleza inaccesibles a la razón, tal vez no hubieran sido nunca descubiertas por ella, si se la hubiera dejado sola. En este horizonte se sitúan cuestiones como el concepto de un Dios personal, libre y creador, que tanta importancia ha tenido para el desarrollo del pensamiento filosófico y, en particular, para la filosofía del ser. A este ámbito pertenece también la realidad del pecado, tal y como aparece a la luz de la fe, la cual ayuda a plantear filosóficamente de modo adecuado el problema del mal. Incluso la concepción de la persona como ser espiritual es una originalidad peculiar de la fe. El anuncio cristiano de la dignidad, de la igualdad y de la libertad de los hombres ha influido ciertamente en la reflexión filosófica que los modernos han llevado a cabo. Se puede mencionar, como más cercano a nosotros, el descubrimiento de la importancia que tiene también para la filosofía el hecho histórico, centro de la Revelación cristiana. No es casualidad que el hecho histórico haya llegado a ser eje de una filosofía de la historia, que se presenta como un nuevo capítulo de la búsqueda humana de la verdad (Juan Pablo II, 1998: n. 76).

Finalmente, nos parece que tanto el contexto de la discusión - entre autores católicos - como la intervención pontificia dejan claro que la filosofía cristiana no es una filosofía inspirada en cualquier interpretación de la revelación cristiana, como, por ejemplo, en el luteranismo o en cualquiera otra de las divisiones del cristianismo. La discusión presupone la verdad de la revelación tal como la Iglesia católica la define y la propone para ser creída con fe teologal. De ahí que otros autores prefieran hablar directamente de «filosofía católica». Aunque esta expresión es menos frecuente, la han usado autores como von Balthasar (1946) y Chesterton (2007). Alasdair MacIntyre, él mismo un converso al catolicismo, asume esta denominación desde la perspectiva de un tomista peculiar, como puede verse en su comentario a Fides et ratio (MacIntyre, 2006). MacIntyre incurre abiertamente en lo que van Steenberghen temía: la afirmación de un contraste neto, al que la fe nos condena o nos libera, entre la filosofía católica -especialmente el realismo tomista- y las filosofías ateas. Sin embargo, MacIntyre evita el peligro de encerrarse en un ghetto o de afirmar que existe esa especie de condena o carga, la de verse forzado a pensar de manera diferente a los demás. Ciertamente habrá esa diferencia o contraste, en la medida en que buena parte del pensamiento moderno se ha apartado del sentido común y del realismo metafísico ejemplificado por Tomás de Aquino (MacIntyre, 2006: 184-196). En este sentido, sin que la fe sea una carga o condena, el diálogo racional entre creyentes y 
no creyentes se convierte en un conflicto de visiones globales en el cual las dos partes deben estar dispuestas a corregir los errores a la luz de la crítica racional y a dar el debido peso a todas las objeciones racionales (MacIntyre, 2006: 187-188). Además, la fe católica no detiene la reflexión ni ahorra las dificultades filosóficas. El filósofo católico no puede detener su cuestionamiento cuando encuentra la certeza de la fe, pues precisamente la revelación le impele a extender más el campo del conocimiento (MacIntyre, 2006: 182-183). Y es precisamente acerca de esta confrontación que el diagnóstico de Alasdair MacIntyre nos parece muy instructivo, aunque no sea demasiado alentador.

\section{El diagnóstico de MacIntyre}

El diagnóstico de la situación actual de la filosofía y en particular de la filosofía católica, que nos ofrece MacIntyre, es, en síntesis, el siguiente. Primero, la tradición de la filosofía católica - aquella que se cultiva en armonía con la fe, influida vitalmente por la revelación divina en sus aspectos subjetivo y objetivo y apoyada por la autoridad magisterial de la Iglesia en cumplimiento de su misión propia- ha estado ausente de los debates filosóficos de la modernidad, con un par de excepciones aisladas, que, según MacIntyre, son Rosmini y Newman (MacIntyre, 2009: 133137; Coreth, 1989: 117-120). Solamente a partir de la Aeterni Patris recomienza la filosofía católica, entendida entonces - por las indicaciones del papa - prácticamente como sinónimo del Tomismo. León XIII estimó que los principales errores de la modernidad eran de carácter filosófico y que debían ser corregidos desde dentro de la filosofía, para lo cual la misma filosofía moderna, que se había apartado de la fe, no contaba con los recursos necesarios. Era imprescindible acudir a Tomás de Aquino (MacIntyre, 2009: 153).

El cultivo del tomismo siguió dos derroteros en tensión: el de la investigación erudita, que se hizo cargo de los problemas, y el de la difusión del pensamiento de santo Tomás en resúmenes y manuales, que muchas veces redujo a fórmulas sencillas un pensamiento abstraído de sus problemas y de los conflictos con las respuestas rivales. Durante el siglo XX, los filósofos católicos desarrollaron diversas versiones del tomismo, pero también filosofías en contraste, al menos parcial, con el tomismo: el realismo crítico de Mercier, la fenomenología de Wojtyla, la filosofía analítica de Anscombe, Geach, Dummett y otros, etc. De manera que, en 1998, Juan Pablo II, reiterando la posición estratégica del Tomismo -especialmente su concepción de la verdad y del hombre como procedente de Dios y dirigido a Dios-, reconoce como parte de la tradi- 
ción de la filosofía católica una historia de conflictos y desacuerdos. MacIntyre repasa la variedad de autores abrazados por Fides et Ratio como modelos de la tradición (Juan Pablo II, 1998: n. 74; MacIntyre, 2006: 192; MacIntyre, 2009: 169). Estos conflictos son posibles, sin embargo, sobre la base de acuerdos profundos subyacentes: los puntos de partida existenciales (i.e., las cuestiones humanas que se plantean las personas corrientes) y la confianza en la razón humana, así como una cierta imagen común del hombre en el cosmos y de Dios (MacIntyre, 2009: 165170).

Segundo, contra la posibilidad de la filosofía católica se yergue la institucionalización de la filosofía secularizada. En realidad, la verdadera filosofía - la que preserva la dimensión sapiencial, que aborda las cuestiones existenciales y ordena todos los saberes humanos desde una perspectiva superior- es la filosofía católica. La audacia del autor alcanza cotas insospechadas, casi insolentes. Esta vera philosophia, sin embargo, no puede ser cultivada como tal en los ámbitos institucionales contemporáneos, ni siquiera en las universidades católicas de primera línea, que se han plegado a los criterios secularizados de lo que significa tener éxito en la empresa académica y filosófica. En efecto, para «la cultura dominante de la modernidad secularizada» (MacIntyre, 2009: 170) —observa MacIntyre, aunque en esto no es original: el malestar es extendido entre los filósofos (Nussbaum, 2010)_, «la filosofía es entendida generalmente como tan solo una forma más de actividad académica especializada» (MacIntyre, 2009: 170-171). Esta actividad es «importante quizás para aquellos cuyos intereses los inclinan hacia esa clase de asunto» (MacIntyre, 2009: 171); pero es también «algo que posee poca relevancia para los asuntos prácticos, algo que puede ser ignorado sin riesgos por la inmensa mayoría de la humanidad, que no es de ninguna manera una parte indispensable de una adecuada educación» (MacIntyre, 2009: 171). La universidad de investigación se ha convertido en una gran corporación de negocios, exitosa en la investigación especializada, en la formación de los profesionales técnicos necesarios para la sociedad del capitalismo avanzado y en la acumulación de riquezas procedentes de esos ámbitos interesados en los resultados prácticos de sus investigaciones y en sus profesionales.

La universidad de investigación ha perdido cualquier sentido de la conexión entre las disciplinas y de la contribución de todas ellas, ordenadamente, al logro de una meta superior a la meramente pragmática: una comprensión compartida del universo por parte de sus profesores y estudiantes. En consecuencia, se trata de una universidad en la que ciertas cuestiones, a saber, las fundamentales, no se preguntan (MacIntyre, 2009: 174-175). También John Finnis ha sostenido que «la estructura fundamental de la universidad moderna [...] promueve la ignorancia y la falsa 
creencia acerca de los hechos más importantes. Y promueve activamente la ignorancia, la falsa creencia y la indiferencia acerca de los más importantes bienes humanos» (Finnis, 2012: 1). Esta exclusión afecta especialmente a la verdadera filosofía y a la teología. La teología «ha sido en la mayor parte de los casos expulsada totalmente de la universidad de investigación» (MacIntyre, 2009: 175), según MacIntyre. A eso cabe añadir que las universidades católicas alcanzan, a lo más, a preservar alguna Facultad o Departamento de Teología que, abrazando los criterios seculares de la excelencia científica - lo que ya constituye un error suicida-, se constituyen en ámbitos aislados de investigación, sin ninguna relevancia para las demás ciencias humanas. La filosofía, por su parte, «ha sido marginada [...] de dos maneras» (MacIntyre, 2009: 175). Por una parte, en el mejor de los casos es tratada como cualquier otra disciplina aislada, y es valorada en la medida en que contribuye a la formación de estudiantes bien preparados para sus carreras profesionales ulteriores; pero «la noción de que los seres humanos necesitan la filosofía, de que la filosofía articula y se mueve hacia dar respuesta a preguntas cuya formulación es crucial para la realización humana, esta noción es totalmente ajena al ethos de la universidad de investigación» (MacIntyre, 2009: 176). Por otra parte, al seguir el modelo de especialización de las otras disciplinas, los filósofos profesionales escriben dentro de, y para un círculo de, especialistas, con un vocabulario semitécnico, y con un dominio de una creciente literatura profesional que tiene solo unos pocos lectores, de manera que ocultan «qué es lo que podría dar a sus elucidaciones alguna importancia más general» (MacIntyre, 2009: 176). De esta manera, según MacIntyre, los filósofos profesionales de este tipo «excluyen con éxito, de la discusión, a todos con excepción de sus colegas. Esos filósofos colaboran inconscientemente con un público filosóficamente ineducado en hacer que la filosofía parezca no solo difícil - que lo es- sino también inaccesible «que no necesita serlo» (MacIntyre, 2009: 176).

\section{Propuesta para ir adelante}

La propuesta de Alasdair MacIntyre, conocedor de la confrontación desde las dos veredas — primero la no católica y ahora la católica_-, es que los filósofos católicos sigan la indicación de Juan Pablo II, es decir, que vuelvan a cultivar la filosofía de tal manera que ella enfrente las preocupaciones humanas básicas, sin sacrificar ni el rigor ni la profundidad (MacIntyre, 2009: 176).

Esta tarea se ha de proseguir confrontando las tradiciones rivales, lo cual les exige, a los filósofos católicos, abordar explícitamente la cuestión de las relaciones entre sus convicciones prefilosóficas (i.e., su adhesión a la 
verdad revelada) y sus investigaciones filosóficas, no solamente para responder a la crítica nietzscheana, sino, sobre todo, porque la misma fe católica les demanda (a ellos, aun cuando no a todos los creyentes) tener buenas razones - no la sola fe- para aceptar ciertas verdades acerca de Dios y de la ley natural (MacIntyre, 2009: 177). La tarea exigirá, además, enfrentar los conflictos al interior de la propia tradición de la filosofía católica (MacIntyre, 2009: 179). Y, puesto que la causa principal del error en la filosofía moderna es la no orientación del hombre hacia Dios, las respuestas racionales de la filosofía católica exigen un diálogo y un debate sobre todo el rango de las preguntas fundamentales sobre Dios, el hombre y el universo (MacIntyre, 2009: 179).

En mi opinión, el planteamiento de MacIntyre es a veces en exceso conflictivista o dialéctico. Carga las tintas sobre la incompatibilidad de las tradiciones rivales de investigación filosófica y de las diversas convicciones ideológicas o simplemente culturales, hasta el punto de que ha podido dar la impresión —errada, tal como yo lo entiendo- de un cierto perspectivismo cultural extremo (MacIntyre, 1992: 267-289). Sin embargo, su propuesta de reforma universitaria implica la posibilidad de ese diálogo racional en el marco de una obligada participación en el conflicto intelectual. Esta tesis es más optimista que la tolerancia aparente en la universidad liberal, que, en el ámbito de la filosofía y las humanidades, aboca a recluirse cada uno, libremente, en sus propias investigaciones.

A partir de su diagnóstico en apariencia pesimista y de su propuesta profundamente optimista de participar en un conflicto continuado, sin evadirlo sino más bien aprovechándolo para progresar en la verdad, MacIntyre sostiene que, ya que la filosofía verdadera implica una ordenación de todos los saberes y su relación con la teología, la investigación filosófica no se puede proseguir en aislamiento de todos esos otros saberes. Y esto exige un marco institucional que es la Universidad, pero no tal como están estructuradas las universidades seculares de investigación o las universidades católicas que las imitan.

De manera que cualquier Universidad Católica en la que tales proyectos fuesen a ser perseguidos exitosamente necesitará poseer estructuras y objetivos muy diferentes a los de las grandes universidades seculares de investigación, y no solamente en razón del lugar central otorgado al estudio de la teología. Tanto sus estudios de grado como los de posgrado, especialmente en filosofía, pero también más en general, serían muy diferentes (MacIntyre, 2009: 179).

Hemos visto ya (sección 2) en qué medida el concepto de filosofía católica, más aún que el de filosofía cristiana, es problemático, pues la Igle- 
sia católica no tiene una filosofía oficial y reconoce a sus fieles un amplio campo de libertad para desarrollar escuelas filosóficas que reconozcan e incorporen, con sentido crítico, cualquier verdad presente en cualquier autor, sin excluir incluso a los expresamente anticatólicos. La tesis de MacIntyre, no obstante, está revestida del peso de quien ha hecho precisamente eso, además de haber transitado vitalmente por varias posiciones filosóficas contrastantes con la fe. El filósofo escocés afirma que la filosofía católica, más allá de la legítima pluralidad de escuelas coherentes con la fe, puede hacer más relevante la filosofía al vincularla con los problemas perennes de todo ser humano, de manera prominente y no solamente cuando los filósofos salen de su laboratorio al acercarse a la muerte. Por ese camino, la filosofía católica vendría también a ser significativa para los debates culturales contemporáneos.

Actualmente, en muchas universidades de prestigio - católicas y no católicas - no se abordan en un nivel filosófico - mediante una argumentación racional rigurosa, profunda y, además, valiente- la mayoría de las cuestiones capitales respecto de las cuales existe un intenso debate en la sociedad civil y en la pugna política. Entonces la filosofía se torna irrelevante, porque renuncia a su rol directivo de las deliberaciones sociales con las que efectivamente se relaciona una comunidad de filósofos.

No intento desconocer el riesgo del activismo político, que subordina el valor de la verdad y de la profundidad al valor del poder y de la inmediatez. Mas existe también el extremo opuesto: un universalismo filosófico desencarnado, que, bajo el pretexto de una especialización científica según el modelo de determinadas ciencias empíricas de ámbito extremadamente local, olvida la misión política de la sabiduría como ciencia rectora de la vida en común. La más eficaz manera de incurrir en ese universalismo desencarnado es evadir también las cuestiones universales que interesan a todos los seres humanos - aun a quienes se desinteresan activamente de la filosofía, por influjo precisamente de una filosofía materialista o pragmatista-, que son precisamente las cuestiones que también se hacen presentes en los debates políticos locales de más acuciante actualidad.

El punto de equilibrio entre los dos extremos o peligros (i.e., la politización contingente de la filosofía y el universalismo abstracto y desencarnado), aunque siempre sea un equilibrio inestable, no debe renunciar a la relevancia de las reflexiones filosóficas para los debates sociales, en los que tanto la fe como la razón y las diversas ideologías o modelos políticos concretos cumplen un rol. Las tradiciones filosóficas, cuando son cultivadas por filósofos católicos influidos vitalmente por la luz de la fe, se incorporan de alguna manera a la matriz de la filosofía católica. En consecuencia, no se trata de una sola filosofía católica, sino de una mul- 
tiplicidad de filosofías parcialmente en concurrencia, pero con presupuestos básicos comunes. Dos rasgos compartidos por cualquier filosofía católica han de ser, en mi opinión, (i) remover los obstáculos, mediante la evitación de los errores más graves sobre esos presupuestos fundamentales (v.gr., sobre la naturaleza de Dios, la dignidad de la persona humana, el sentido de su vida, la espiritualidad e inmortalidad del alma, las exigencias éticas fundamentales...), y (ii) proseguir positivamente el impulso a plantear y a hacerse cargo de las preguntas filosóficas que realmente angustian o preocupan al hombre qua hombre.

La valentía de pronunciarse sobre esas cuestiones fundamentales en sede filosófica puede ser el próximo desafío que haga salir a la filosofía católica de su presente irrelevancia.

\section{REFERENCIAS}

-Balthasar, H. U. von (1946). Von den Aufgaben der Katolischen Philosophie in der Zeit. Einsiedeln: Johannes Verlag.

-Benedicto XVI (2005). Carta Encíclica Deus caritas est, 25 de diciembre.

-Bréhier, E. (1988). Historia de la filosofía (Vol. 1). Madrid: Tecnos.

-Chesterton, G. K. (2007). El Pozo y los Charcos. Buenos Aires: Agape Libros.

-Coreth, E. et al. (1989). La filosofía del siglo XX. Barcelona: Herder.

-Derisi, O. (1979). Concepto de la filosofía cristiana. Buenos Aires: Club de Lectores.

-Dworkin, R. (2013). Religion Without God. Cambridge: Harvard University Press.

-Finnis, J. (2012). ¿Acaso la universidad corrompe a la juventud? El Derecho, 13.141: 1-4.

-Finnis, J. (2013). Ruptura, transformación y continuidad en la tradición de la razón y la justicia. Pro manuscripto, Santiago de Chile.

-García López, J. (1999). La filosofía cristiana en la Fides et Ratio. Anuario Filosófico, 32: 641-662.

-Gilson, E. (2009). El espiritu de la filosofía medieval. Madrid: Rialp.

-Guitton, J. (1998). Mi testamento filosófico. Madrid: Encuentro.

-Juan Pablo II (1998). Carta Encíclica Fides et ratio, 14 de septiembre.

-MacIntyre, A. (1992). Tres versiones rivales de la ética. Madrid: Rialp.

-MacIntyre, A. (2006). Philosophy recalled to its tasks: a Thomistic reading of Fides et Ratio, en The Task of Philosophy. Selected Essays (vol. 1; pp. 179-196). New York: Cambridge University Press.

-MacIntyre, A. (2009). God, Philosophy, Universities: A Selective History of the Catholic Philosophical Tradition. Lanham: Rowman \& Littlefield.

-Maritain, J. (1933). De la philosophie chrétienne. Paris: Desclée de Brouwer.

-Nussbaum, M. (2010). Not for Profit: Why Democracy Needs the Humanities. Princeton: Princeton University Press.

-Ratzinger, J. (2008). Lo que cohesiona al mundo. Los fundamentos morales y prepolíticos del Estado liberal, en J. Habermas \& J. Ratzinger: Entre razón y 
religión. Dialéctica de la secularización (pp. 35-54). México D.F.: Fondo de Cultura Económica.

-van Steenberghen, F. (1993). Filosofía y Cristianismo. Scripta Theologica, 25(3): 1087-1092.

Sumario: Introducción; 1. La controversia centenaria sobre la filosofía cristiana; 2. El diagnóstico de MacIntyre; 3. Propuesta para ir adelante; Referencias. 